\title{
Comparative Analysis of the Electromyography Activity of Core Muscles During Balance Pad- and Sling-assisted Exercises
}

\author{
Yaoyao Liu', MSc, Won-gyu Yoo², PhD, Su-Jung Kim³, PhD \\ ${ }^{1}$ Department of Physical Therapy, Graduate School of Inje University, ${ }^{2}$ Department of Physical Therapy, College of Health Care Medical \\ Science and Engineering, Inje University, Gimhae, ${ }^{3}$ KEMA Healing Center, Busan, Korea
}

\author{
Article Info \\ Received October 26, 2020 \\ Revised October 29, 2020 \\ Accepted October 30, 2020 \\ Corresponding Author \\ Won-gyu Yoo \\ E-mail:won7y@inje.ac.kr \\ https://orcid.org/0000-0001-6200-9674
}

\section{Key Words}

Core muscle

Electromyography

Sling exercise

Unstable surface
Background: Unstable surface-based core training can significantly enhance core strength, but no studies have compared the effects of balance pad- and sling-assisted exercises.

Objects: To study the effects of unstable surface-based balance pad- and sling-assisted core strength exercises on muscle activity.

Methods: Twenty male students aged 20-25 years participated in this study. The effects of three types of core strength exercises, performed with a sling or balance pad, on the activities of three muscles, i.e., the right musculus obliquus externus abdominis (EO), right erector spinae (ES), and right gluteus maximus (GM), were examined.

Results: 1) In the glute bridge exercise, the percentage of maximum voluntary contraction of the EO, ES, and GM were significantly different between the balance pad- and sling-assisted exercises. The relative contribution of the ES and GM activities to all muscle activity were not significantly different between the two training types, whereas that for EO showed a significant difference. 2) There was no significant difference in the percentage value of maximum voluntary contraction (\%MVC) among the EO, ES, and GM during the "leg-lifting with flat support"exercise, and there were no significant difference in the relative contributions between the two training types. 3) In the "side bridge leg separation exercise", the \%MVC of the $E S, E O$, and GM were significantly different between the two training types.

Conclusion: Sling training for core muscles was generally better than balance pad assist training. The majority of physiotherapy patients require core training. Our results could guide physiotherapists in the choice of targeted exercises for these patients.

\section{INTRODUCTION}

The core is integral to almost all bodily movements [1]. The core encompasses the waist, pelvis, and hip joints [2-4]. The lumbopelvic hip complex provides a strong and stable base for all movements [5,6]. To maintain stability, the center of gravity should generally be within the core area when performing activities. All types of forces to which the human body is exposed during exercise are transmitted to the core area through the support surface, and then to all of the points at which power is generated. The core produces, transmits, and controls power during sports.

In daily life, the body is often in motion, such as walking, running, jumping, etc.; stability is very important under such dynamic conditions. There have been a number of studies on core functions. A stable core provides a foundation for strong dynamic muscle contractions $[7,8]$. Core strength exercises mainly target control of the main muscle groups at the center of the body, which in turn control other muscles to provide a stable center of gravity and balance. Strengthening the core muscles has been advocated to prevent and rehabilitate various lumbar spine and musculoskeletal disorders, and enhance athletic performance [9]. Chung et al. [10] reported that core training improved balance (via its effects on the lumbar-pelviship complex) and posture; dynamic balance ability can be improved by shifting the center of gravity. Anderson et al. [11] compared the electromyographic (EMG) activity produced by push-ups performed on stable and unstable surfaces: instability in the upper and lower body, and trunk muscle activation, increased on the unstable surface. 
Limb movements emanate from the trunk, which plays a decisive role in ensuring balance and stability of the spine and pelvis. Physical fitness exercises can be divided into five major types: traditional core, core stability, ball/balance device, free weight, and noncore free weight exercises [12]. Balance exercises can be considered as a type of core stability training, because these exercises activate the core muscles. Sudden perturbations applied to the body can shift the center of gravity outside the base of support. To avoid losing balance and falling, postural adjustments are made to move the center of gravity back within the base of support [13]. Sling exercise training (SET) is a common core training method targeting tissues and muscles via some simple or complicated movements, which improves core control [14-16]. Studies have shown that exercises involving an unstable surface, such as Swiss exercise ball and Dynair Ballkissen balance pad exercises, can improve posture stability and center of gravity control $[17,18]$.

There have been many studies on core training methods, and on physical fitness recovery and balance control in clinical patients. Technical evaluations and analyses of sports performance, muscle injury and fatigue have also been performed. However, few clinical studies have compared unstable surfacebased training performance with sling versus balance pad assistance. Therefore, this study aimed to provide reference surface EMG (sEMG) data for these two training types, to facilitate treatment planning by physiotherapists.

\section{MATERIALS AND METHODS}

\section{Participants}

The study population consisted of 20 healthy males with no history of back pain in the previous year, recruited from among the student population of our university [19]. Volunteers were required to have engaged in regular physical exercise over the past 6 months, and were excluded if they participated in sports at an elite level, had a history of musculoskeletal complaints in the past 6 months, or had any contraindications to exercise [20]. Participants with a body mass index $\geq 25$ were excluded due to the potential influence of fatty tissue on our ability to measure sEMG activity [21]. The participants had a mean age of $23.6 \pm 1.27$ years (range: $20-25$ years), mean height of $179.3 \pm 3.29 \mathrm{~cm}$, and mean weight of $73.35 \pm 4.82 \mathrm{~kg}$. All participants provided written informed consent in accordance with a protocol approved by the Human Ethics Committee of the Institute of Health Sciences, Inje University.

\section{Instrumentation}

The sEMG data were collected using 4D Molecular Therapeutics (4D-MT) software (4D-Molecular Therapeutics, Emeryville, CA, USA). Use LT-302-3035 $(30 \times 35 \mathrm{~mm})$ foam electrode sheet, including $4.0 \mathrm{~mm}$ buckle core.

The ratio of left-handed to right-handed people worldwide is about 3:17, which indicates that the right muscle dominates most people's daily life. Therefore, we chose the right side of the body as the target side of the experiment (all the included volunteers were right handedness). The electrode plate was placed on the skin covering the muscle bellies of interest, i.e., the right musculus obliquus externus abdominis (EO), right erector spinae (ES), and right gluteus maximus (GM). The skin was shaved and cleaned with rubbing alcohol, and the electrodes were attached with an interelectrode distance of $20 \mathrm{~mm}$ [22]. The sEMG electrode attachment positions were as follows: right EO: the apophysis of the $\mathrm{EO}, \sim 10 \mathrm{~cm}$ from the navel, aligned with the direction of muscle fibers; right ES, at the level of the twelfth thoracic vertebra and first lumbar vertebra at the "bulge" of the ES; right GM, at the most prominent part of the muscle belly, aligned with the muscle fibers [23].

\section{Procedures}

Before the experiment, volunteers were allowed to warm-up for 10 minutes, to reduce the risk of muscle damage during the test. The following exercises were then performed:

1) Glute bridge exercise: the participants were in a supine position, with the knees bent at $90^{\circ}$, feet placed on a balance pad a comfortable distance apart, and arms flat against the sides of the body. The hips were lifted using the abdominal and buttock muscles. For the sling-assisted exercise, the feet were placed in the sling with the feet and shoulders in the same plane.

2) "Leg-lifting with flat support" exercise: both elbows were bent and supported by the mat. The shoulder joint was perpendicular to the elbow joint, the supporting leg was straight, the supporting foot was placed on the balance pad, and the active leg gradually lifted the body in the sagittal plane. For the sling-assisted exercise, the feet were placed in the sling with the feet and shoulders in the same plane.

3) "Side bridge leg separation exercise": the subject lay on their side with the elbow joint of the supporting arm bent and 
the knee joint of the supporting leg placed on the balance pad. The hip was lifted as much as possible, and the active leg was gradually lifted to the horizontal plane. For the sling-assisted exercise, the feet were placed in the sling, with the feet and elbows kept in the same plane.

To ensure the reliability, the planes of motion and fulcrum were kept consistent as far as possible (Figure 1).

A linear relationship between muscle tension and sEMG signal amplitude has been reported [11]. Therefore, sEMG amplitude can be used as a measure of muscle activity, by normalizing the EMG signal relative to the maximum voluntary contraction (MVC) [24]. To test muscle activity in this study, the maximum EMG activity (EMGmax) of the muscle during MVC was measured. In previous studies, a percentage value (\%EMGmax) was used as the basic measure of muscle activity [25-27]. Prior to the MVC test in this study, the subjects were asked to exert submaximal isometric force during each exercise prior to the actual test procedure. After the warm- up and 3 minutes rest period, the subjects were instructed to progressively increase the force exerted up to the maximum force, taking about 5 seconds twice with at least 3 minutes between trials to rule out fatigue effects. The trial with the highest peak force was analyzed [22]. The MVC procedure was based on VeraGarcia et al. [28].

\section{Data analysis}

The 4D-Molecular Therapeutics software was used to save the EMG data to the computer. The data were normalized relative to those obtained during MVC of each muscle (\%EMGmax), as a standardized value similar to previous studies [22]. The activities of each muscle were analyzed and compared based on the percentage of maximum voluntary contraction (\%MVC). The activity of each muscle relative to the overall muscle activity was also determined.

SPSS software (ver. 20.0; IBM Co., Armonk, NY, USA) was used for the statistical analysis. All variables had a normal distribution. Therefore, the independent samples t-test was used to compare GM, ES, and EO muscle activities between the balance pad and sling training types, for all three exercises. In all analyses, $\mathrm{p}<0.05$ was taken to indicate statistical significance.
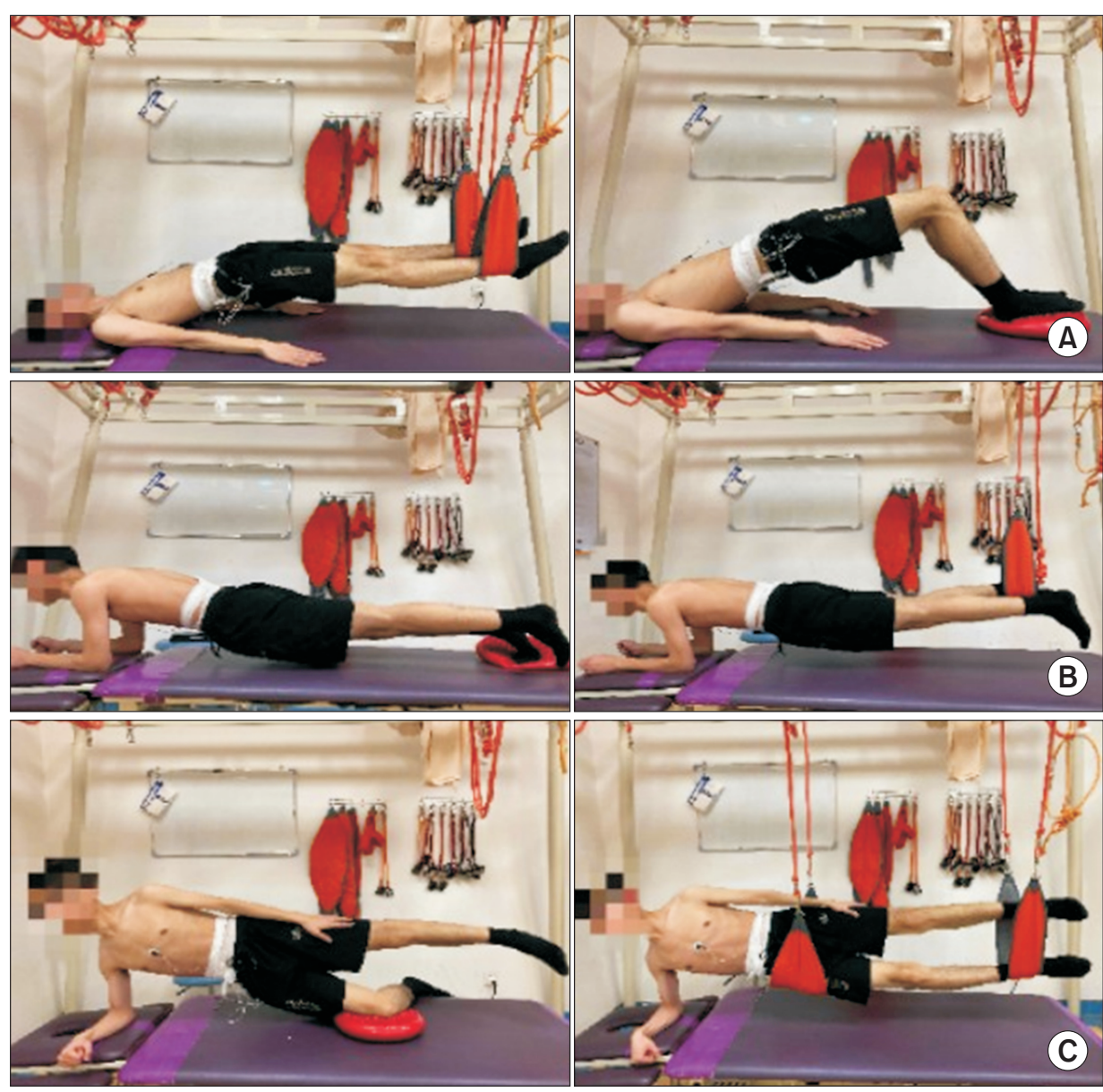

Figure 1. (A) Glute bridge exercise. (B) Leglifting with flat support exercise. (C) Side bridge leg separation exercise. 


\section{RESULTS}

Tables 1-3 show the EMG activity data of the 20 volunteers, for all sling-assisted and -unassisted exercises. Regarding the glute bridge exercise, the GM had the highest \%MVC and the EO had the lowest \%MVC. The \%MVC values of the EO, ES, and GM were significantly different between the balance padand sling-assisted exercises (all, $\mathrm{p}<0.05$ ). The activity of each muscle as a proportion of the overall activity during the glute bridge exercise was also calculated; the relative contributions of ES and GM activity to overall muscle activity did not differ between two training methods, whereas that of EO showed a significant difference $(\mathrm{p}<0.01)$. These observations were in line with our expectations that the glute bridge exercise would elicit significant ES and GM activity, and that sling-assisted training performance would be superior to balance pad-assisted performance.

Regarding the leg-lifting with flat support exercise, the EO had the highest \%MVC and the ES had the lowest \%MVC. There were no significant differences in the \%MVC values of the EO, $\mathrm{ES}$, or GM between the balance pad- and sling-assisted exercises (all, $p>0.05$ ). The relative contributions of the different muscles to overall muscle activity did not differ between the two training types (all, $\mathrm{p}>0.05$ ).

Regarding the side bridge leg separation exercise, the \%MVC values of the EO and ES were higher when the sling was used compared to the sling-unassisted exercise. The \%MVC of the ES was significantly different between the two training types ( $\mathrm{p}$ $=0.016)$, as were those of the EO and GM $(\mathrm{p}<0.01)$. Furthermore, the \%MVC values of the EO GM differed significantly $(\mathrm{p}<$

Table 1. Statistical analysis of glute bridge exercise data $(\mathrm{N}=20)$

\begin{tabular}{|c|c|c|c|c|c|c|}
\hline \multirow{3}{*}{ Mode } & \multicolumn{6}{|c|}{ Muscle } \\
\hline & \multicolumn{2}{|c|}{ EO } & \multicolumn{2}{|c|}{ ES } & \multicolumn{2}{|c|}{ GM } \\
\hline & Balance pad & Sling & Balance pad & Sling & Balance pad & Sling \\
\hline$\% M V C$ ASD & $15.85 \pm 5.68$ & $20.67 \pm 8.06$ & $34.58 \pm 7.63$ & $40.71 \pm 10.28$ & $46.75 \pm 10.94$ & $56.00 \pm 13.46$ \\
\hline$p$-value & \multicolumn{2}{|c|}{0.035} & \multicolumn{2}{|c|}{0.039} & \multicolumn{2}{|c|}{0.022} \\
\hline РоT \%MVC & $32.53 \pm 9.02$ & $17.90 \pm 6.70$ & $55.54 \pm 5.80$ & $32.72 \pm 6.40$ & $47.85 \pm 8.01$ & $47.39 \pm 6.28$ \\
\hline
\end{tabular}

Values are presented as mean \pm standard deviation. EO, musculus obliquus externus abdominis; ES, erector spinae; GM, gluteus maximus; ASD, average standard deviation; PoT \%MVC, percentage of total \%MVC.

Table 2. Statistical analysis of leg-lifting with flat support exercise data $(N=20)$

\begin{tabular}{|c|c|c|c|c|c|c|}
\hline \multirow{3}{*}{ Mode } & \multicolumn{6}{|c|}{ Muscle } \\
\hline & \multicolumn{2}{|c|}{ EO } & \multicolumn{2}{|c|}{ ES } & \multicolumn{2}{|c|}{ GM } \\
\hline & Balance pad & Sling & Balance pad & Sling & Balance pad & Sling \\
\hline $\begin{array}{l}\% \text { MVC ASD } \\
\text { p-value }\end{array}$ & $44.18 \pm 10.55$ & $45.06 \pm 9.94$ & $18.55 \pm 9.77$ & $22.09 \pm 8.08$ & $22.50 \pm 9.30$ & $23.80 \pm 9.21$ \\
\hline $\begin{array}{l}\text { PoT \%MVC } \\
\text { p-value }\end{array}$ & $52.45 \pm 9.32$ & $50.24 \pm 9.55$ & $21.28 \pm 8.97$ & $23.90 \pm 6.59$ & $26.28 \pm 9.50$ & $25.86 \pm 8.38$ \\
\hline
\end{tabular}

Values are presented as mean \pm standard deviation. EO, musculus obliquus externus abdominis; ES, erector spinae; GM, gluteus maximus; ASD, average standard deviation; PoT \%MVC, percentage of total \%MVC.

Table 3. Statistical analysis of side bridge leg separation exercise data $(\mathrm{N}=20)$

\begin{tabular}{|c|c|c|c|c|c|c|}
\hline \multirow{3}{*}{ Mode } & \multicolumn{6}{|c|}{ Muscle } \\
\hline & \multicolumn{2}{|c|}{ EO } & \multicolumn{2}{|c|}{ ES } & \multicolumn{2}{|c|}{ GM } \\
\hline & Balance pad & Sling & Balance pad & Sling & Balance pad & Sling \\
\hline$\% M V C A S D$ & $36.95 \pm 9.24$ & $51.37 \pm 12.73$ & $23.89 \pm 8.43$ & $31.39 \pm 10.24$ & $49.79 \pm 9.59$ & $39.44 \pm 9.80$ \\
\hline $\mathrm{p}$-value & \multicolumn{2}{|c|}{0.000} & \multicolumn{2}{|c|}{0.016} & \multicolumn{2}{|c|}{0.002} \\
\hline РоT \%MVC & $33.46 \pm 5.44$ & $41.90 \pm 6.58$ & $21.27 \pm 5.41$ & $25.49 \pm 6.60$ & $45.29 \pm 5.25$ & $32.61 \pm 6.89$ \\
\hline p-value & \multicolumn{2}{|c|}{0.000} & \multicolumn{2}{|c|}{0.033} & \multicolumn{2}{|c|}{0.000} \\
\hline
\end{tabular}

Values are presented as mean \pm standard deviation. EO, musculus obliquus externus abdominis; ES, erector spinae; GM, gluteus maximus; ASD, average standard deviation; PoT \%MVC, percentage of total \%MVC. 
0.01). The relative contributions of the EO, GM, and ES muscle activities to overall muscle activity were significantly different between the two different training methods $(\mathrm{p}<0.01, \mathrm{p}<0.01$, and $\mathrm{p}<0.05$, respectively).

\section{DISCUSSION}

In this study, two different training methods, i.e., balance pad- and sling-assisted, were used during the glute bridge, leg-lifting with flat support, and side bridge leg separation exercises. EMG activity was measured in the right EO, right ES, and right GM core muscles. In a previous study, sling-assisted rollout and "body-saw" exercises generally elicited at least 60\% MVC of the rectus abdominis and $\mathrm{EO}$, consistent with our data [29].

The EMG activity in all abdominal muscles during the gluteal bridge exercise increased significantly when Both Sides Up and Swiss exercise balls were used [30]. In the present study, the gluteal bridge exercise elicited the highest GM activity among the exercises. However, this was not the case for the abdominal muscles, consistent with previous studies. Muscle activation was higher during sling- versus balance pad-assisted exercises.

Although the exercises were not the same as the present study, a previous study indicated that the muscle activities of the ipsilateral GM, erector spinae, and contralateral latissimus dorsi muscle on the unstable side, i.e., the side with the hip joint extended, were increased by the prone hip extension exercise [31]. Another study show that in a variety of flat support motions, in sling training for ankle support, the activity of trunk muscles was significantly increased [32]. In this study, during the leg-lifting with flat support exercise, the unstable side was supported by an unstable surface. This had an obvious effect on abdominal muscle activation. Although the slingassisted performance was better than the balance pad-assisted performance, the back and hip muscles on the supported side were still not activated effectively due to the unstable surface. In an experiment similar to the present study, Comfort et al. [33] showed that the bridge exercise can effectively increase the activity of abdominal muscles, but not the paravertebral muscles.

We hypothesized that muscle activities would be higher during sling- versus balance pad-assisted exercises, but for the side bridge leg separation exercise, GM activity, and the relative contribution thereof to overall muscle activity, were higher when the balance pad was used; the opposite was true regarding the EO and ES activities. One possible explanation for these findings is that the knee and ankle joints are supported during balance pad- and sling-assisted training, respectively, where changes in support type change GM muscle activity (because other muscles around the GM may share the load).

This study had several limitations. First, the sample size was small; a larger sample would yield more reliable results. Second, activation of other muscles may have interfered with the experiment. Third, although the supporting surfaces and bodily support points were kept consistent as far possible, slight differences may have had an impact on the data. Fourth, the current study only included male volunteers, without the participation of female volunteers. Due to these limitations, further studies are required.

\section{CONCLUSIONS}

This study was performed to compare the effects of sling and balance pad assistance on core muscle activities during three exercises. With the sling, the activity of the EO and GM muscles, but not the ES muscle, was greater. The majority of physiotherapy patients require core training. Our results could guide physiotherapists in the choice of targeted exercises for these patients.

\section{CONFLICTS OF INTEREST}

No potential conflict of interest relevant to this article was reported.

\section{AUTHOR CONTRIBUTIONS}

Conceptualization: WY. Data curation: YL. Formal analysis: YL. Investigation: YL. Methodology: YL, SJK. Resources: YL. Supervision: WY. Validation: WY, SJK. Visualization: WY. Writing - original draft: YL. Writing - review \& editing: WY, SJK.

\section{ORCID}

Yaoyao Liu, https://orcid.org/0000-0002-2522-8402

Su-Jung Kim, https://orcid.org/0000-0002-2586-7378 


\section{REFERENCES}

1. Cabanas-Valdés R, Bagur-Calafat C, Girabent-Farrés M, Caballero-Gómez FM, Hernández-Valiño M, Urrútia Cuchí G. The effect of additional core stability exercises on improving dynamic sitting balance and trunk control for subacute stroke patients: a randomized controlled trial. Clin Rehabil 2016;30(10): 1024-33.

2. Willson JD, Dougherty CP, Ireland ML, Davis IM. Core stability and its relationship to lower extremity function and injury. J Am Acad Orthop Surg 2005;13(5):316-25.

3. Hasegawa I. The use of unstable training for enhancing sport performance. NSCA Perform Train J 2005;4(4):15-7.

4. Pilates JH. Your health: a corrective system of exercising that revolutionizes the entire field of physical education. Incline Village: Presentation Dynamics; 1998.

5. Leetun DT, Ireland ML, Willson JD, Ballantyne BT, Davis IM. Core stability measures as risk factors for lower extremity injury in athletes. Med Sci Sports Exerc 2004;36(6):926-34.

6. Kibler WB, Press J, Sciascia A. The role of core stability in athletic function. Sports Med 2006;36(3):189-98.

7. Brumitt J, Matheson JW, Meira EP. Core stabilization exercise prescription, part I: current concepts in assessment and intervention. Sports Health 2013;5(6):504-9.

8. Huxel Bliven KC, Anderson BE. Core stability training for injury prevention. Sports Health 2013;5(6):514-22.

9. Akuthota V, Nadler SF. Core strengthening. Arch Phys Med Rehabil 2004;85(3 Suppl 1):S86-92

10. Chung EJ, Kim JH, Lee BH. The effects of core stabilization exercise on dynamic balance and gait function in stroke patients. J Phys Ther Sci 2013;25(7):803-6.

11. Anderson GS, Gaetz M, Holzmann M, Twist P. Comparison of EMG activity during stable and unstable push-up protocols. Eur J Sport Sci 2013;13(1):42-8.

12. Martuscello JM, Nuzzo JL, Ashley CD, Campbell BI, Orriola JJ, Mayer JM. Systematic review of core muscle activity during physical fitness exercises. J Strength Cond Res 2013;27(6): 1684-98.

13. Willardson JM. Core stability training: applications to sports conditioning programs. J Strength Cond Res 2007;21(3):97985.

14. Chen L, Chen J, Peng Q, Chen J, Zou Y, Liu G. Effect of sling exercise training on balance in patients with stroke: a metaanalysis. PLoS One 2016;11(10):e0163351.
15. Gaedtke A, Morat T. TRX suspension training: a new functional training approach for older adults - development, training control and feasibility. Int J Exerc Sci 2015;8(3):224-33.

16. Petrofsky JS, Batt J, Davis N, Lohman E, Laymon M, De Leon GE, et al. Core muscle activity during exercise on a mini stability ball compared with abdominal crunches on the floor and on a Swiss ball. J Appl Res 2007;7(3):255-72.

17. Romero-Franco N, Martínez-López E, Lomas-Vega R, HitaContreras F, Martínez-Amat A. Effects of proprioceptive training program on core stability and center of gravity control in sprinters. J Strength Cond Res 2012;26(8):2071-7.

18. Lee D, Kim H, An H, Jang J, Hong S, Jung S, et al. Comparison of postural sway depending on balance pad type. J Phys Ther Sci 2018;30(2):252-7.

19. Vera-Garcia FJ, Elvira JL, Brown SH, McGill SM. Effects of abdominal stabilization maneuvers on the control of spine motion and stability against sudden trunk perturbations. J Electromyogr Kinesiol 2007;17(5):556-67.

20. Mok NW, Yeung EW, Cho JC, Hui SC, Liu KC, Pang CH. Core muscle activity during suspension exercises. J Sci Med Sport 2015;18(2):189-94.

21. Park SY, Yoo WG. Effect of height of feet on trunk muscle activity and pelvic tilt angle during prone bridge exercises. Isokinet Exerc Sci 2016;24(3):189-94.

22. Maeo S, Takahashi T, Takai Y, Kanehisa H. Trunk muscle activities during abdominal bracing: comparison among muscles and exercises. J Sports Sci Med 2013;12(3):467-74.

23. Yuan JT. [Experimental study on the effect of three bridge exercises on superficial core muscle of male college students]. Chengdu, Chengdu Institute of Physical Education, Master Dissertation. 2017. Chinese.

24. Petrofsky JS, Prowse M, Bains GS, Sharma A, Batt J, Gunda S. Core muscle use in superficial and deep abdominal muscles with a crunchless abs video. J Appl Res 2009;9(3):88-99.

25. Petrofsky JS. Frequency and amplitude analysis of the EMG during exercise on the bicycle ergometer. Eur J Appl Physiol Occup Physiol 1979;41(1):1-15.

26. Petrofsky JS, Lind AR. Frequency analysis of the surface electromyogram during sustained isometric contractions. Eur J Appl Physiol Occup Physiol 1980;43(2):173-82.

27. Petrofsky JS. Computer analysis of the surface EMG during isometric exercise. Comput Biol Med 1980;10(2):83-95.

28. Vera-Garcia FJ, Moreside JM, McGill SM. MVC techniques to normalize trunk muscle EMG in healthy women. J Electro- 
myogr Kinesiol 2010;20(1):10-6.

29. Cugliari G, Boccia G. Core muscle activation in suspension training exercises. J Hum Kinet 2017;56:61-71.

30. Czaprowski D, Afeltowicz A, Gębicka A, Pawłowska P, Kędra A, Barrios C, et al. Abdominal muscle EMG-activity during bridge exercises on stable and unstable surfaces. Phys Ther Sport 2014;15(3):162-8.

31. Kim SY, Chae JB, Kwon JH. Physical therapy session duration in patients with low back pain: descriptive Research. J Korean
Acad Orthop Man Ther 2001;7(1):51-66.

32. Bak J, Shim S, Cho M, Chung Y. The effect of plank exercises with hip abduction using sling on trunk muscle activation in healthy adults. J Korean Phys Ther 2017:29(3):128-34.

33. Comfort P, Pearson SJ, Mather D. An electromyographical comparison of trunk muscle activity during isometric trunk and dynamic strengthening exercises. J Strength Cond Res 2011;25(1):149-54. 